\title{
Vesicular stomatitis virus modified with single chain IL-23 exhibits oncolytic activity against tumor cells in vitro and in vivo
}

This article was published in the following Dove Press journal:

International Journal of Interferon, Cytokine and Mediator Research

20 May 2010

Number of times this article has been viewed

James M Miller'

Sarah McNulty Bidula ${ }^{1,5}$

Troels Mygind Jensen ${ }^{1,6}$

Carol Shoshkes Reiss $1,2,3,4$

'Department of Biology, New York University, New York, NY, USA;

${ }^{2}$ Center for Neural Science, NYU;

${ }^{3} \mathrm{NYU}$ Cancer Institute; ${ }^{4}$ Departments

of Microbiology, NYU School of

Medicine and Mt Sinai School

of Medicine, New York, NY, USA;

${ }^{5}$ Present address Graduate Program,

University of Pittsburgh School

of Medicine, Pittsburgh, PA,USA

${ }^{6}$ Present address: Univercity of

Copenhagen, Copenhagen, Denmark

Correspondence: Carol Shoshkes Reiss Department of Biology, New York University, 100 Washington Sq East, Silver Center room 1009, New York, NY, 10003-6688, USA

$\mathrm{Tel}+1212-998-8269$

Fax+1212-995-40I5

Email carol.reiss@nyu.edu

\begin{abstract}
Viruses are potentially attractive agents for development as novel oncolytic agents. Reverse genetic approaches allow for the attenuation of candidate viruses and can enhance their ability to exploit inherent cellular and molecular properties of tumors, including deficiencies in interferon (IFN) signaling. Vesicular stomatitis virus (VSV) is a promising oncolytic agent for exactly these reasons. VSV infection of immunocompetent mice is usually rapidly cleared due to the virus' sensitivity to type I IFN responses. However, in tumors that are unable to activate the IFN response, VSV is able to replicate without inhibition, resulting in cell destruction. Unfortunately, when VSV is introduced into mice intranasally or systemically via therapeutic doses into tumor-bearing rodents, hosts may develop fatal encephalitis. We have previously found that a recombinant VSV expressing the pro-inflammatory cytokine interleukin-23 (IL-23) is significantly attenuated in the central nervous system (CNS). As a result of this, we hypothesized that attenuation in the CNS is partially a result of enhanced NO response as a result of IL-23 signaling. Infection of the CNS with this virus (designated VSV23) is characterized by decreased viral replication, morbidity, and mortality. We have now extended those studies which reveal that VSV23 maintains oncolytic capacity in vitro in multiple cell lines including NB41A3 neuroblastomas, L929 adipose-derived cells, immortalized BHK-21 cells, and the murine mammary derived JC cells. Additionally, in vivo VSV23 infection results in JC tumor destruction and induces enhanced memory responses against tumor cells.
\end{abstract}

Keywords: oncolysis, IL-23, vesicular stomatitis virus, tumor cells, oncolytic activity

Cancer is the second leading cause of death in the United States. Breast cancer is the most common malignancy diagnosed in women, and is responsible for approximately 41,000 deaths annually, inclusive of men. ${ }^{1}$ Metastasis is most often seen in the bone, lung, live, and brain. Traditional chemotherapy has been shown to have very low efficacy in treating these metastases and, as such, the need for novel treatments is clear.

Adenoviruses and retroviruses were originally used as oncolytic agents, however these both have severe limitations including toxicity and germ-line viral integration. Second generation oncolytic viruses including Sindbis, Newcastle disease virus (NDV), herpes simplex virus-1 (HSV-1), and vesicular stomatis virus (VSV) are currently under development as cancer therapies. ${ }^{2-5} \mathrm{VSV}$ is particularly promising as it selectively destroys a wide variety of tumor cell lines including those with aberrant p53, Ras, or Myc function such as glioblastomas, colorectal, pancreatic, and breast cancer cells. ${ }^{6-12}$ Other targets of VSV therapy include leukemia, hepatocellular carcinoma, nasopharyngeal carcinoma, and bladder cancer. ${ }^{8,13-20}$ Mutations in tumor cells allow unchecked replication within an 
organism, as they render many tumors unresponsive to immune responses including the interferon (IFN) response pathways. VSV preferentially replicates in tumor cells specifically due to deficiencies in the antiviral IFN pathways of these cells. ${ }^{6,21}$ In the case of VSV, most humans are seronegative, and as such do not have preexisting memory responses that could interfere with the efficacy of therapeutic treatment. ${ }^{22,23}$ This, combined with the high replication rate of VSV in tumor cells, provides ample opportunity for VSV to induce significant tumor destruction before adaptive immune responses eliminate the viral infection. $^{24}$

Recombinant VSVs (rVSVs) are of particular interest as researchers are able to take advantage not only of the preferential tumor replication, but are also able to engineer the virus to enhance the oncolytic capability. A recombinant VSV expressing the $\mathrm{F}$ protein from NDV inducing syncytia in infected cells has been used to treat hepatocellular carcinoma in a rat model. ${ }^{25}$ Another rVSV, with a mutation in the M protein (VSV(M51R)LacZ) has shown promise in a mouse model of metastatic breast cancer. ${ }^{7}$ VSV expressing the GFP protein (VSVGFP) has shown the ability to lyse several colorectal cancer cell (CRC) lines including MCA26, SW-480, and HT-29. ${ }^{8} \mathrm{VSV}$ expressing interleukin (IL)-12 has been tested in the mouse and rat models of breast and colorectal cancer. ${ }^{26}$ Other experiments have indicated that selective replication-competent VSVs may be a treatment option for glioma. ${ }^{27}$

A major advantage of using VSV as an oncolytic treatment is its ability to infect and destroy cells under hypoxic conditions. Solid tumors are generally not homogenous, and as such are characterized by multiple microenvironments. The hypoxic environment is a barrier for effective treatment of tumors by traditional radiation or chemotherapies. VSV has been shown both in vitro and in vivo to be able to overcome hypoxia-induced inhibition of RNA and protein synthesis through increased RNA production and increasing eIF-2 $\alpha$ phosphorylation respectively. ${ }^{28}$

Another possibility for increasing the efficacy of VSV is to enhance antitumor immunity. It is likely that no matter how effective viral treatment is, some transformed cells will escape viral mediated destruction. In this event, antigen presentation by tumors may not elicit an immune response and the tumor will be reestablished. Studies conducted with vaccinia infected lysates have shown that antigen presentation by infected tumor cells results in an increased antitumor immune response. ${ }^{29}$ In addition, it is possible that virus-induced apoptosis of tumor cells could result in the presentation of apoptotic cell fragments by host dendritic cells, which may be enhanced via induction of Toll-like receptors by viral RNAs, leading to a proinflammatory state. ${ }^{30-32}$

We hypothesized that the proinflammatory cytokine IL-23 would be an ideal candidate to improve the both the direct oncolytic capacity of VSV and the host's antitumor immune responses. IL-23 is a heterodimeric member of the IL-12 family of cytokines composed of a p40 subunit shared with IL-12 and a unique $\mathrm{p} 19$ subunit. A heterodimeric receptor IL-23R includes a unique chain and the IL-12R $\beta 1$ subunit. ${ }^{33}$ IL-23R is expressed on the surface of immune cells including T cells, NK cells, macrophages, and dendritic cells. Binding of IL-23 to IL-23R results in signaling through the Jak, Tyk, STAT pathways, and the transcription factor RoR $\gamma \mathrm{T} .{ }^{34,35} \mathrm{IL}-23$ is produced by macrophages and dendritic cells. ${ }^{36}$ IL-23 also plays a role in the differentiation of proinflammatory T cells secreting IL-17. ${ }^{37}$

Antimetastatic and antitumor properties have been associated with IL-23 and IL-12, however the therapeutic use of IL-12 in cancer treatment has been compromised by side effects associated with the induction of IFN- $\gamma^{38-43}$ The antitumor effects associated with IL-23 are attributed to production of TNF- $\alpha$ (not IFN- $\gamma$ ), and result in enhanced effectiveness of $\mathrm{CD}^{+} \mathrm{T}$ cells. ${ }^{44}$ Experiments indicated that depletion of NK cells had no affect on the antitumor activity of IL-23 as determined by tumor growth rate, however $\mathrm{CD}^{+} \mathrm{T}$ cell depletion was found to significantly compromise the ability of naive mice to reject implanted tumors and limit tumor growth rates. ${ }^{45}$ Experiments conducted with IL-23-transduced CT26 cells and B16F1 tumor cells have shown increased anti-tumor memory $\mathrm{T}$ cell responses and decreased lung metastases in vivo. ${ }^{41}$ Experiments conducted with the murine mammary cancer cell line MA891 have shown that expression of IL-23 through retrovirus transduction resulted in increased anti-tumor CTL effectors. ${ }^{46}$

In previous research we have reported the successful production, characterization, and safety of VSV23. VSV23 has also been shown to induce robust innate and adaptive immune responses. We also previously found that VSV23 was capable of infecting and destroying the murine mammary derived JC tumor line in vitro. ${ }^{47}$ We report here that VSV23 is capable of infecting and killing multiple tumors, can induce tumor-specific immune responses, and can exhibit enhanced tumor destruction over other recombinant control viruses.

\section{Materials and methods Cell lines}

BHK-21 baby hamster kidney cells, JC murine mammary gland adenocarcinoma-derived cells, L929 murine adipocytes, 
and NB41A3 murine neuroblastoma cells were purchased from the American Type Culture Collection (Manassas, VA). BHK-21 cells were grown in minimum essential media (Mediatech, Manassas, VA) with 1\% nonessential amino acids (NEAA), 1\% penicillin-streptomycin (penstrep), and 10\% fetal bovine serum (FBS), JC cells grown in RPMI1640 (Mediatech) with 1\% pen-strep and 10\% FBS, L929 cells grown in Dulbecco's modified of Eagle's medium (Mediatech) with 1\% pen-strep, 1\% HEPES buffer, 1\% L-glutamine, and 10\% FBS, NB41 A3 grown in F-12K media (Mediatech) with $2.5 \mathrm{FBS}$ and $15 \%$ horse serum.

\section{Viruses}

Three recombinant viruses (VSV23, VSVST, and VSVXN2) were produced for this project utilizing the reverse genetics method originally described by the Rose lab. ${ }^{47,48}$ Briefly, VSV23 contains a single chain IL-23 (scIL-23) comprised of the p40 and p19 subunits joined by a flexible linker. Cells infected with VSV23 produce bioactive IL-23. VSVST and VSVXN2 were produced to control for attenuation of VSV resulting from alterations in genome size and particle morphology. VSVST was generated by mutagenizing the p40 subunit of IL-23, resulting in three stop codons. Infection with this virus does not result in production of IL-23. VSVXN2 is a recombinant minimal genome VSV that contains no additional gene insertions.

\section{In vitro viral infection}

JC cells and BHK-21 cells were grown to $70 \%$ confluence in $10 \mathrm{~cm}$ tissue culture dishes; cells were infected with VSV23, VSVST, VSVXN2, or VSVwt at MOI $=1.0$ or $\mathrm{MOI}=0.1$ (respectively) and incubated for 8 hours at $37^{\circ} \mathrm{C}$ and $5 \% \mathrm{CO}_{2}$. Digital photographs were then taken using an Olympus BH2-RFCA microscope (Olympus, Center Valley, PA). Uninfected cells were used as controls. Upon detection of cytopathic effect (CPE), $10 \mu \mathrm{L}$ of supernatant from each group was then transferred to an individual well of L929 cells that had been grown to $70 \%$ confluence. Samples were incubated overnight at $37^{\circ} \mathrm{C}$ and $5 \% \mathrm{CO}_{2}$. A total of $10 \mu \mathrm{L}$ of supernatant from each group was again transferred to an individual well of NB41 A3 cells that had been grown to $70 \%$ confluence. Cells were visually monitored for signs of CPE for 8 hours and digital photographs were taken.

\section{Animal studies}

All procedures involving animals were approved by and performed according to the guidelines of The University Animal Welfare Committee of New York University. Eight to ten-week-old male BALB/cAnNTac (BALB/c) mice were purchased from Taconic Farms, Inc. (Germantown, NY), housed under standard conditions and fed ad libitum. Mice were housed at NYU for one week prior to initiation of experiments.

\section{Tumor implantation and viral treatment}

Cohorts of $N=4$-8-to 10 -week-old male BALB/c mice were injected subcutaneously on the left dorsal flank with $1 \times 10^{7} \mathrm{JC}$ cells suspended in $40 \mu \mathrm{L}$ sterile HBSS. Animals were monitored for solid tumor development. Ten days post-implantation, tumors were injected with $1 \times 10^{7}$ plaquefolming unit (PFU) of VSV23, VSVST, or VSVXN2 diluted in $40 \mu \mathrm{L}$ of PBS or vehicle alone. Viral treatments were repeated on days 3 and 5 after the initial treatment. All viral doses were delivered to four distinct quadrants of the tumor. Tumors were measured daily using electronic calipers and tumor size was calculated using the equation: (length/2) $)^{2}$ (width) ${ }^{2}$.

\section{Immunohistochemical staining (IHC)}

Fourteen days after viral treatment was initiated, animals were arterially perfused with Hank's buffered saline solution (HBSS) and tumors were surgically removed. Whole tumors were frozen in Tissue-Tek OCT compound (Sakura, Torrance, CA), sliced into $18 \mu \mathrm{m}$ thick sections using the Leica CM1850UV Cryostat (Leica, Bannockburn, IL) and placed on poly-L-lysine-coated slides. Sections were fixed in 5\% paraformaldehyde for 10 minutes. The sections were then washed twice with PBS and incubated in $20 \mu \mathrm{g} / \mathrm{mL}$ goat anti-mouse IgG (Jackson Immunoresearch Laboratories Inc., West Grove, PA) for 45 minutes when necessary. Sections were then incubated in phosphate-buffered saline (PBS) with Blotto for 45 minutes. The slides were once again washed with PBS and incubated overnight in primary antibodies. Slides were then washed with PBS and incubated in secondary antibody for 45 minutes. After incubation with a secondary antibody, the sections were washed with PBS and mounted with Vectorshield Mounting Medium (Vector Laboratories, Burlingame, CA). Antibodies used are shown in Table 1. Slides were covered with number 1.5 cover slips (Fisher; Waltham, MA), and viewed using a Leica SP5 confocal microscope at 400x magnification.

\section{Cytolytic T lymphocyte (CTL) assay}

Fourteen days after viral treatment was initiated, spleens from individual mice were harvested, teased into a single cell suspension, and resuspended in MEM, 10\% FBS, 1\% Pen-Strep. JC cells were used as target cells and plated in 
Table I Primary and secondary antibodies

\begin{tabular}{|c|c|c|c|c|}
\hline Primary antibody & Specificity & Dilution & Secondary & Dilution \\
\hline Rat $\alpha$-mouse CDIIb & Mouse macrophages & $1: 200$ in PBS & Goat $\alpha$ - rat Alexa Fluor ${ }^{\circledR} 488$ & I: 100 in PBS \& Blotto \\
\hline Rat $\alpha$-mouse RB68C5 & Mouse neutrophils & $1: 200$ in PBS & Goat $\alpha$ - rat Alexa Fluor ${ }^{\circledR} 488$ & I:100 in PBS \& Blotto \\
\hline Rat $\alpha$-mouse L3T4 & Mouse CD4 cells & $1: 200$ in PBS & Goat $\alpha$ - rat Alexa Fluor ${ }^{\circledR} 488$ & $\mathrm{I}: 100$ in PBS \& Blotto \\
\hline Rat $\alpha$-mouse Ly-2 & Mouse CD8 cells & $1: 200$ in PBS & Goat $\alpha$ - rat Alexa Fluor ${ }^{\circledR} 488$ & I:100 in PBS \& Blotto \\
\hline
\end{tabular}

Abbreviation: PBS, phosphate-buffered saline.

96 well V-bottom plates at $1 \times 10^{4}$ cells per well. Responder cells from individual mice of each treatment group were added to target cells in triplicate at effector to target ratios of 100:1, 50:1, 25:1, and 12:1. Plates were centrifuged for 5 minutes at $200 \mathrm{x}$ g to improve cell contact and incubated for four hours at $37^{\circ} \mathrm{C}, 5 \% \mathrm{CO}_{2}$. The CytoTox $96^{\mathrm{TM}}$ nonradioactive cytotoxicity kit (Promega, Madison, WI) was used per the manufacturer's instructions to determine $\mathrm{T}$ cell mediated cytolytic activity. Colomeric results were detected with a Biorad 550 series microplate reader (Biorad, Hercules, CA) at $490 \mathrm{~nm}$. Results are representative of three replicate experiments.

\section{Statistical analysis}

GraphPad Prism statistical software (GraphPad Software Inc., La Jolla CA) was used for ANOVA and Student's $t$-test statistical analyses.

\section{Results}

\section{In vitro viral infection}

Previously we have shown that VSV23 is capable of infecting, and subsequently inducing apoptosis in, the mammary derived JC cell line. ${ }^{47}$ Here we provide digital images of JC cells exhibiting CPE. A major advantage of using VSV as a cancer treatment is the ability of the virus to infect a wide variety of tumor cells. In order to determine if VSV23 maintains this capacity, BHK21 cells were grown to $70 \%$ confluence in vitro in $10 \mathrm{~cm}$ tissue culture dishes and infected at $\mathrm{MOI}=0.1$ with VSV23. Cells were incubated at $37^{\circ} \mathrm{C}$ overnight and examined for evidence of apoptosis. Significant CPE was noted and $10 \mu \mathrm{L}$ of virally infected supernatant was transferred to L929 adipocytes cells grown to $70 \%$ confluence in $10 \mathrm{~cm}$ tissue culture dishes. L929 cells were incubated overnight and CPE was detected. A total of $10 \mu \mathrm{L}$ of virally infected supernatant was transferred to NB41A3 neuroblastoma grown to $70 \%$ confluence in $10 \mathrm{~cm}$ tissue culture dishes. NB41A3 cells were incubated 8 hours, at which time initial signs of CPE were noted and photographed (Figure 1). RT-PCR and Western blot analysis detected VSV M mRNA and VSV G and M proteins in VSV23-infected cell lysates (data not shown). Taken together, we show that CPE in tumors, in vitro, was associated with VSV23 infection. The experiment was repeated with control viruses in order to determine their suitability for future experiments with similar results.

\section{In vivo tumor treatment}

Results of our previous work showed that VSV23 was attenuated in the mouse model and maintained its oncolytic capacity in vitro. ${ }^{47}$ To test whether this capacity remained intact in vivo and whether or not it was enhanced by the expression of IL-23, we treated solid JC tumors with VSV23 and the control viruses, VSVST and VSVXN2. Tumors treated with VSV23 exhibited a reduction in tumor size through the first 6 days of monitoring after treatment was initiated (Figure 2). The average size of VSV23-treated tumors began to increase beyond the original measurement 8 days after treatment was initiated. In one case a tumor was reduced to a size that could not be measured, however this near complete remission lasted only 2 days. Tumors treated with control viruses exhibited decreased growth rates compared to mock-treated tumors during the first 10 days after treatment, however they did not decrease in size from the initial measurement. By the end of the 14-day monitoring period control virus-treated tumors were of similar size to untreated tumors, while VSV23infected tumors remained significantly smaller than untreated tumors $(P<0.005)$. There were no cases of complete tumor regression detected in any of the treatment groups.

\section{IHC}

Immune responses against viral infection and tumor cells result in a variety of immune cell recruitment. Hypothetically, immune cell infiltration of tumors may be altered by VSV23 infection, due to the secretion of the cytokine. To test this, tumors were isolated 14 days after initiation of viral treatments. Tumors were sectioned and probed for macrophages, neutrophils, $\mathrm{CD}^{+} \mathrm{T}^{+}$, and $\mathrm{CD} \mathrm{T}^{+}$cells. It was not possible to quantitate infiltrating cells due to differences among tissue sections. VSV23-treated tumors appeared to have similar inflammatory cell responses when compared to tumors treated with PBS or other viruses. Analysis of slides using confocal microscopy 


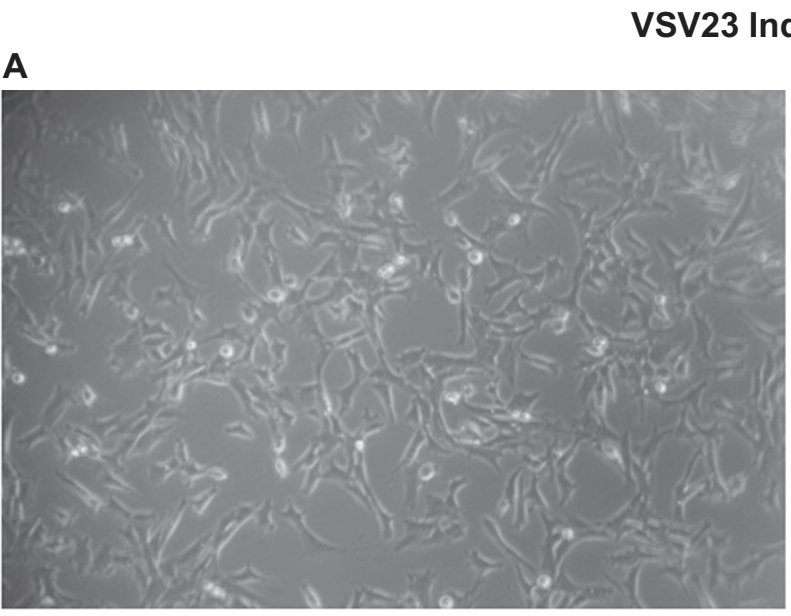

\section{B}

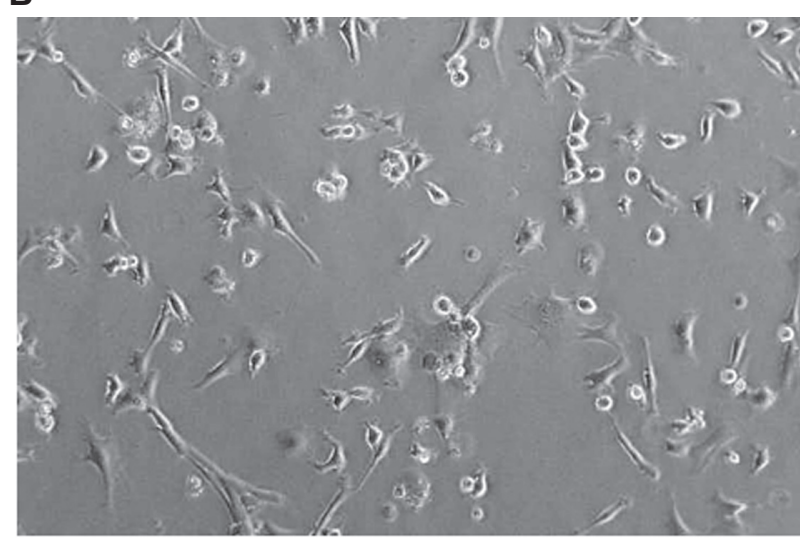

C

D
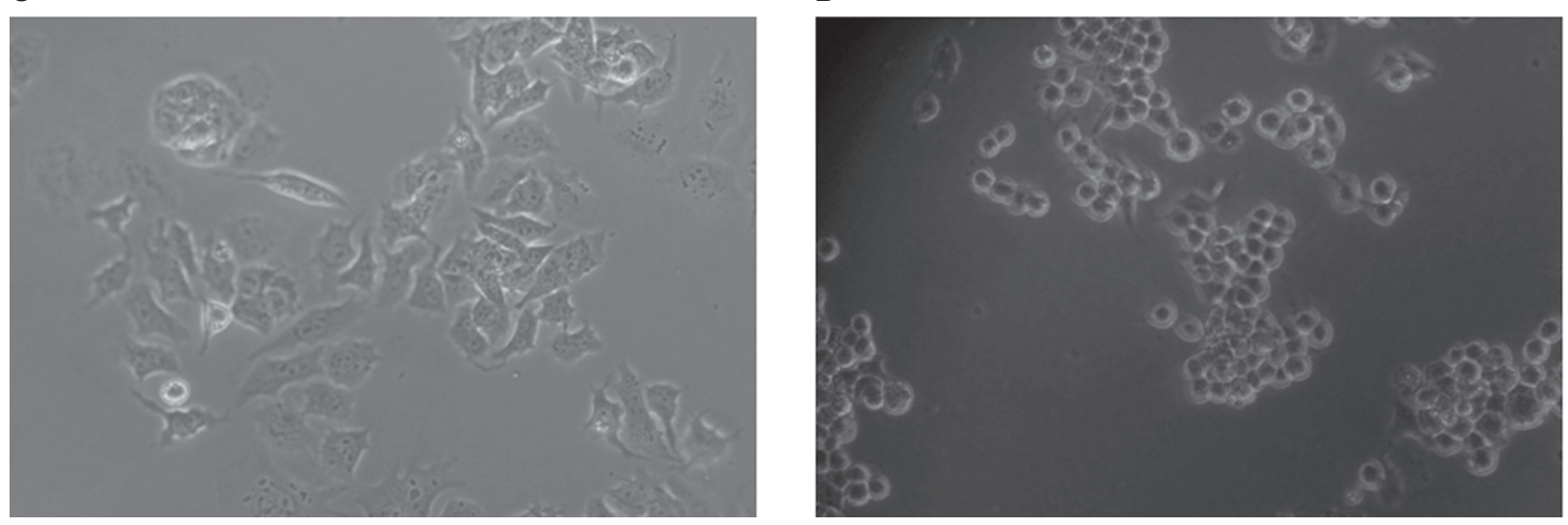

Figure I VSV23 induces CPE and cell death in multiple tumor lines in vitro: A: uninfected JC cells; B: VSV23 infected; C and D: uninfected and VSV23-infected NB4IA3. Images of cells at 8 hours post infection were acquired on an Olympus BH2-RFCA microscope (Olympus, Center Valley, PA) at I00x (A and B) and 200x (C and D).

indicated that all four cell types were recruited to tumors across the panel of viral treatments and mock infection (Figure 3).

\section{Induction of antitumor-specific cytolytic T lymphocytes (CTLs)}

The ability of viruses to elicit host CTL responses which control infection and promote recovery is a hallmark of host acquired immunity to infection. In the case of tumors, CTL responses are induced at varying degrees of robustness and efficacy. In order to determine whether virally induced IL-23 (vIL-23) enhances CTL responses against tumor cells, splenocytes were harvested from tumor bearing animals 14 days after initiation of treatment. Splenocytes were then co-cultured with target JC cells and cell death was measured via a colorimetric assay. This experiment indicated that VSV23 was capable of inducing JC-specific memory CTLs and that the response is more robust than those induced in mock-treated tumors, $P<0.05$ (Figure 4). Additionally, no statistical difference was detected among control viruses and mock treatment.

\section{Discussion}

The need to develop more medical options for treating breast cancer (and in fact all cancers) is clear. Oncolytic viruses represent an exciting opportunity to develop an entirely new class of cancer treatments that will be used in conjunction with traditional modes of therapy. Modern molecular biological approaches further enhance the potential of viral treatments by providing a means of attenuating otherwise harmful viruses and enhancing their innate anti-tumor activities. VSV is a particularly attractive oncolytic agent due to its capacity to infect many tumor lines, the low level of seropositivity in the general population, and the simplicity of its genome, which readily allows for alteration through reverse genetics approaches. ${ }^{21-23} \mathrm{~A}$ variety of transgenes have been introduced into the VSV genome in order to alter the oncolytic efficacy, including the F protein from Newcastle disease virus and IL-12. ${ }^{25,26}$

While VSV infection in humans tends to be asymptomatic, unfortunately infection of tumors in the mouse model at 


\section{Effect of viral treatment on tumor size}

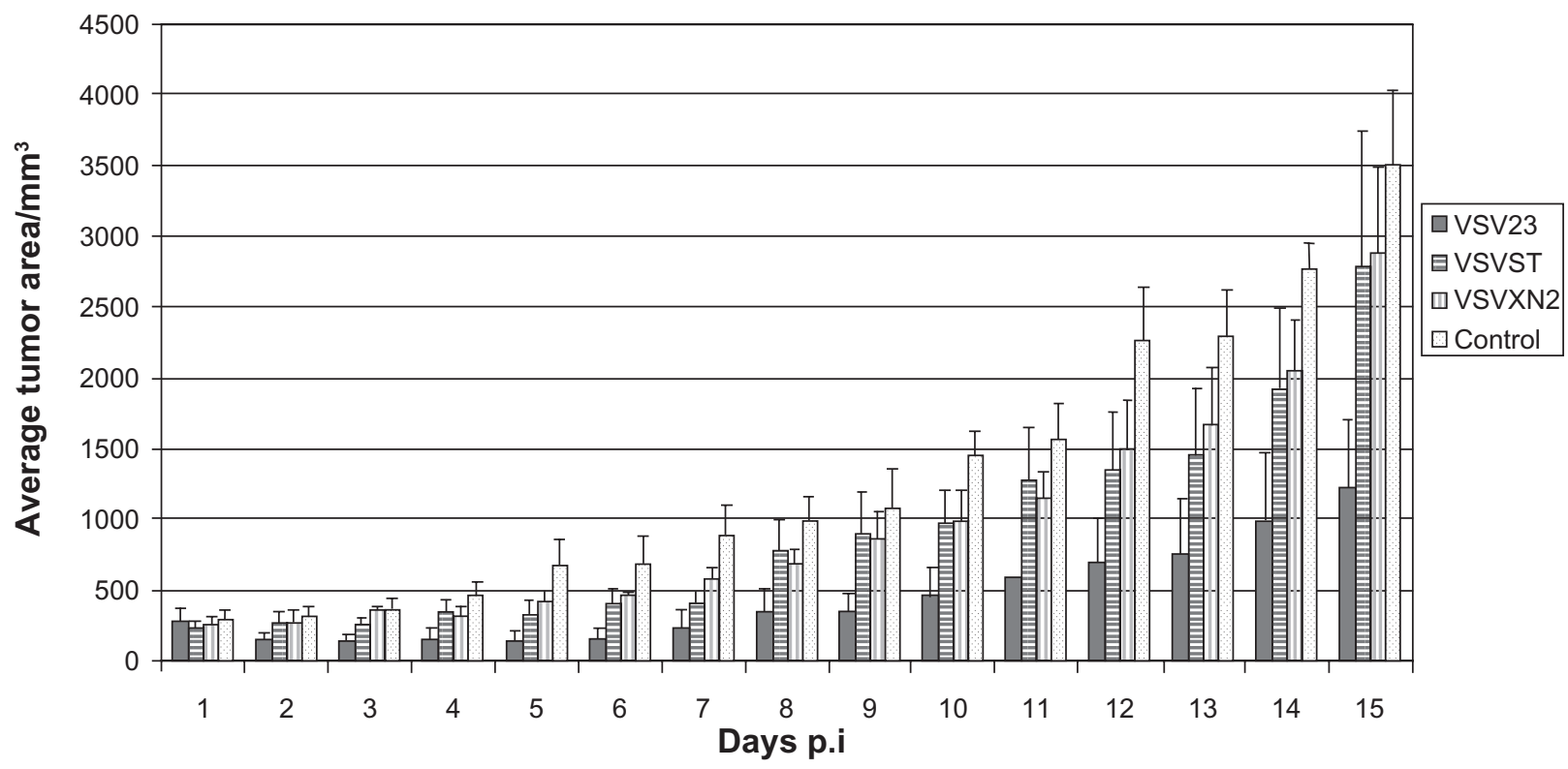

Figure 2 VSV23 infection inhibits tumor growth in vivo. Cohorts of $N=4,8$ to I0-week-old male BALB/c mice were injected subcutaneously on the left dorsal flank with $\mathrm{I} \times 10^{7} \mathrm{JC}$ cells suspended in $40 \mu \mathrm{L}$ sterile HBSS. Ten days post-implantation, tumors were injected with I $\times 10^{7}$ PFU of VSV23 (solid), VSVST (horizontal stripes), VSVXN2 (vertical stripes) diluted in $40 \mu \mathrm{L}$ of PBS or vehicle alone (spotted). Viral treatments were repeated on days 3 and 5 after the initial treatment. VSV23-treated tumors decrease in size during the first six days of monitoring after treatment. The average size of VSV23 treated tumors increases 8 days after treatment. Tumors treated with control viruses were of decreased size compared to untreated tumors through the first 5 days of monitoring. By the end of the I4-day monitoring period control virus-treated tumors were of similar size to untreated tumors, while VSV23-infected tumors remained significantly smaller than untreated tumors $(P<0.005)$. Data shown are representative of three replicate experiments and error bars represent standard deviation.

Abbreviations: HBSS, Hank's buffered saline solution; PDS, phosphate-buffered saline; PFU, plaque-forming unit.

therapeutic doses has been associated with encephalitis and death. ${ }^{6,7}$ Our solution to this problem was to attenuate VSV with the insertion IL-23 into the viral genome resulting in a virus that induces both adaptive and innate immune responses that we designated VSV23. ${ }^{47} \mathrm{VSV} 23$ infection of the CNS is characterized by decreased morbidity and mortality as well as decreased viral titers compared to control and wild-type virus. ${ }^{47}$ Inoculation with VSV23 in the periphery resulted in activation of NK cells, as well as robust adaptive immune responses against the virus, including $\mathrm{CD} 4^{+} \mathrm{T}$ and $\mathrm{CD} 8^{+} \mathrm{T}$ cell recruitment and induction of neutralizing antibodies. ${ }^{47}$ IL-23 was chosen not only for its proinflammatory actions in the CNS, but also for its innate antitumor and antimetastatic properties which are associated with downstream TNF- $\alpha$ induction and enhanced effectiveness of $\mathrm{CD}{ }^{+} \mathrm{T}$ cells. ${ }^{44}$

After determining that VSV23 was immunogenic and attenuated in the CNS we set out to test its oncolytic capabilities. First we conducted an experiment to assess whether VSV23 remained infectious after multiple cell passages and if it was infectious in tumor lines other than JC cells. This was important as alterations to the viral genome might have resulted in defective particles that were only able to carry out one infectious cycle. Such a virus would be unsuitable for in vivo experiments due to negligible tumor destruction. Additionally, the promiscuity of VSV in regards to tumor line infection is one of its key attributes as an oncolytic agent. Alterations to the viral genome could have affected this characteristic of the virus. Serial infections were conducted in three separate tumor lines: BHK21 hamster derived fibroblasts, L929 mouse derived adipocytes, and NB41A3 mouse-derived neuroblastomas (Figure 1). This experiment showed that VSV23 remained infectious after multiple cell passages and that its promiscuity was not abrogated by insertion of IL-23. Therefore VSV23 is suitable for testing in the same tumor lines as are being studied with VSVwt, specifically those deficient in IFN signaling. It should also be noted that many tumors are not homogenous cell populations and as such it is important that oncolytic agents not be restricting to individual cell profiles. From these experimental results we extrapolate that VSV23 will be able to infect most cells in a heterogeneous population.

We next set out to determine the potential efficacy of VSV23 infection in treating breast cancer. Previously we have demonstrated the ability of VSV23 to induce apoptosis in murine mammary derived JC cells in vitro. Here we present data showing the ability of VSV23 to reduce tumor volume and decrease tumor growth rates after multiple 
A

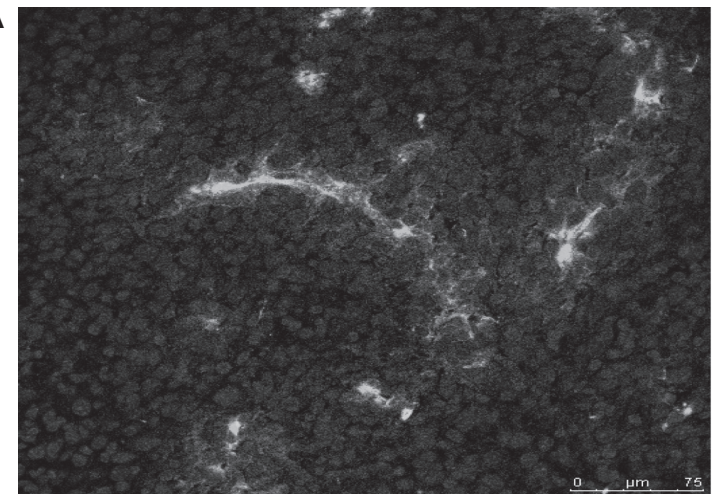

C

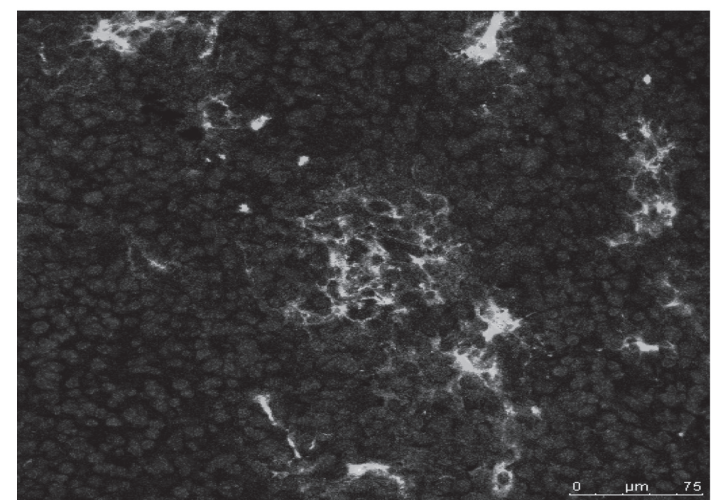

E
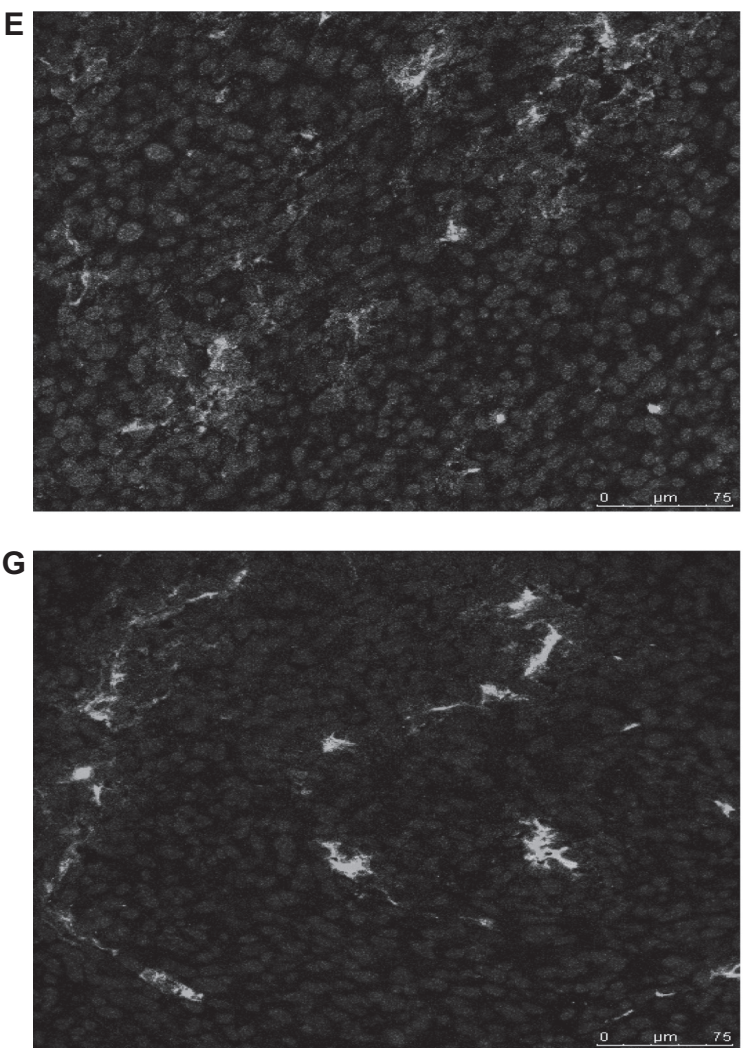

B
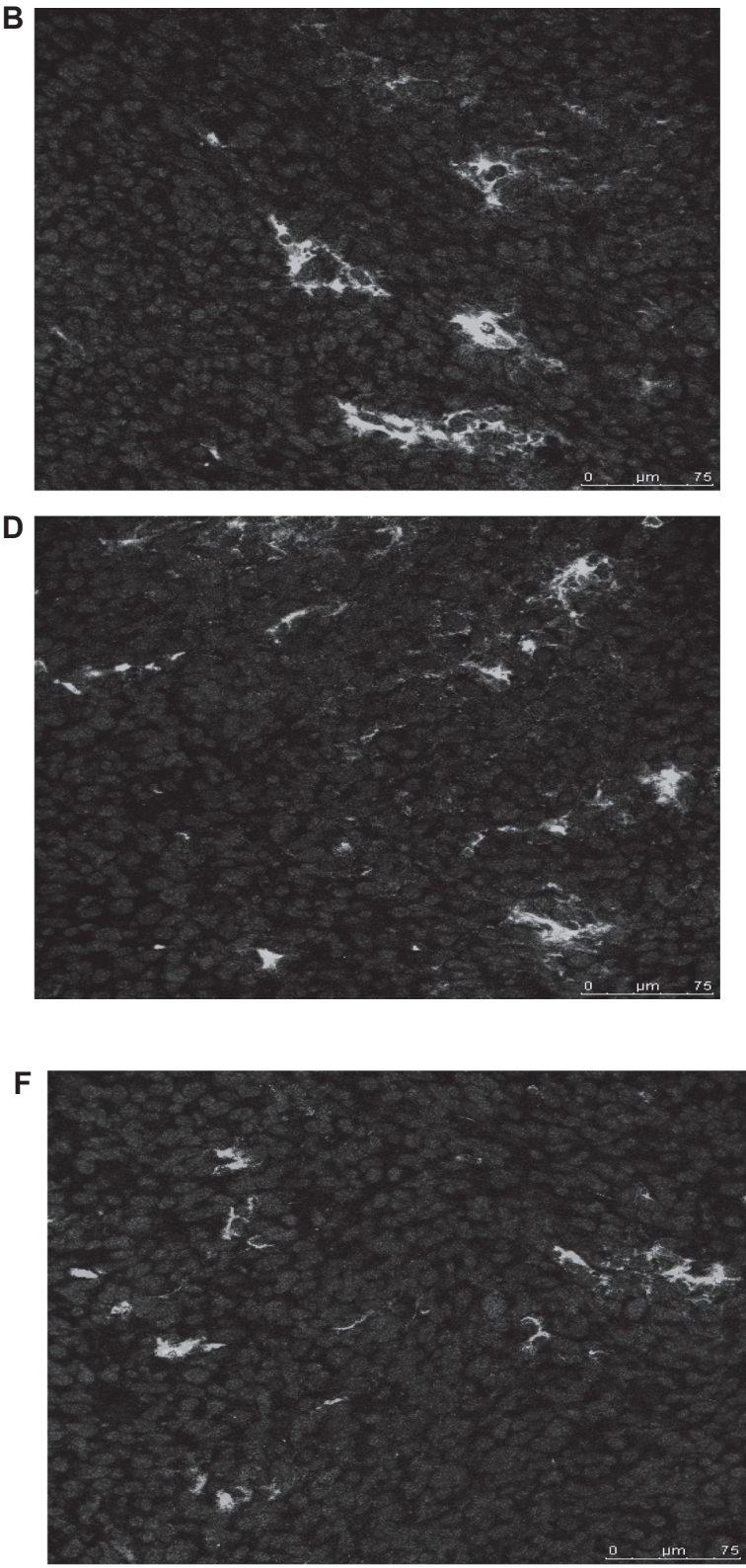

$\mathrm{H}$

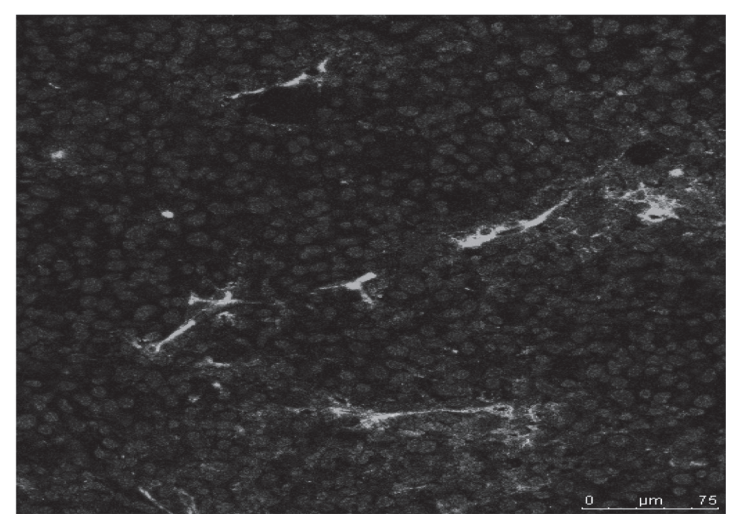

Figure 3 Inflammatory cells infiltrate VSV23 (A, C, E, F) and control rVSV (B, D, F, G) treated tumors. A, B: CD8 ${ }^{+}$T cells. C, D: CD4+ T cells. D, E: Macrophages. F, G: Neutrophils. Cohorts of $N=4,8$ to 10 -week-old male BALB/c mice were injected subcutaneously on the left dorsal flank with I $\times 10^{7} \mathrm{JC}$ cells. Ten days post-implantation, tumors were injected with $\mathrm{I} \times 10^{7}$ pfu of VSV23, VSVST, or VSVXN2 or vehicle alone. Viral treatments were repeated on days 3 and 5 after the initial treatment. Fourteen days after treatment was initiated, tumors were harvested, frozen, sliced into $18 \mu \mathrm{m}$ thick sections, and treated with antibodies specific for cell types. Images were obtained using a Leica SP5 confocal microscope at 400x magnification. Control rVSV-treated tumor images are representative of VSVST andVSVXN2 treated tumors. 


\section{Tumor targeted CTL response}

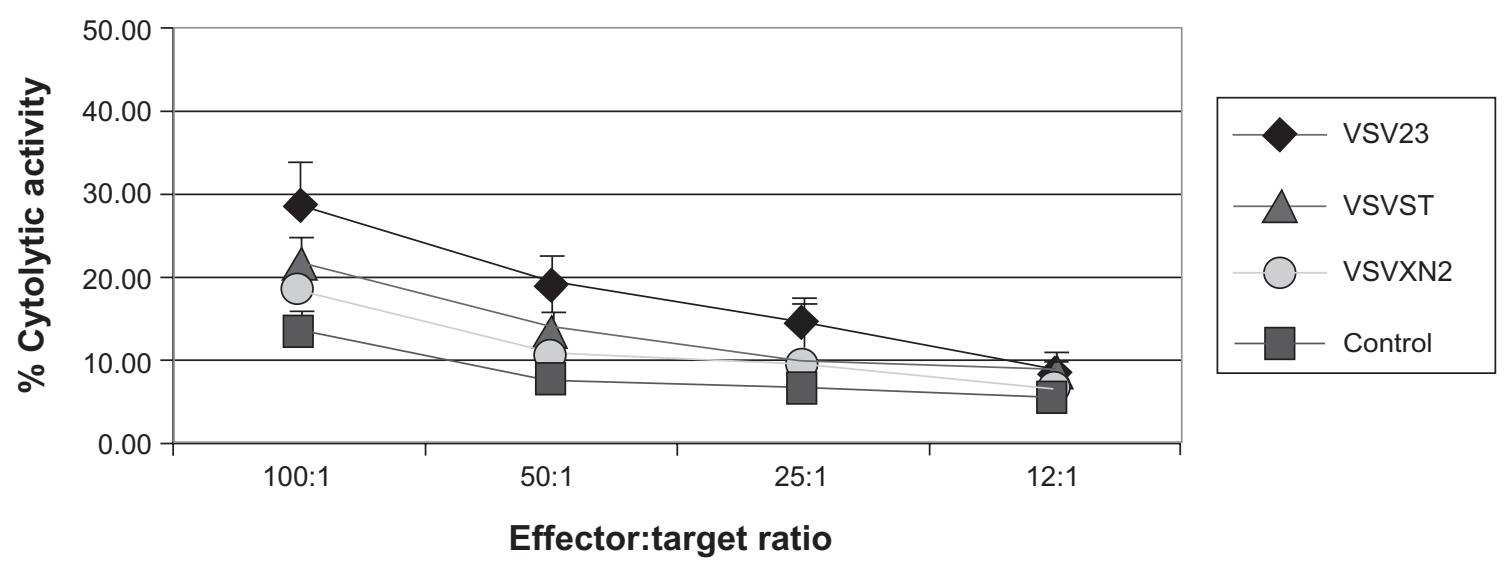

Figure 4 VSV23 treatment results in enhanced memory CTL responses against JC tumor cells in vitro. Cohorts of $N=4,8$ to I0-week-old male BALB/c mice were injected subcutaneously on the left dorsal flank with $\mathrm{I} \times 10^{7} \mathrm{JC}$ cells. Ten days post-implantation, tumors were injected with I $\times 10^{7}$ pfu of VSV23 (diamond), VSVST (triangle), or VSVXN2 (circle), or vehicle alone (square). Viral treatments were repeated on days 3 and 5 after the initial treatment. Then, 14 days after treatment was initiated, splenocytes were harvested and JC cells were cultured in vitro. Cultured naïve T cells from nontumor-bearing animals were used as a negative control. Splenocytes from all tumor-bearing animals exhibited T cell responses against tumor cells, however splenocytes from VSV23-treated animals exhibited enhanced JC tumor-killing capacity. Data presented are means \pm standard deviation and are representative of three replicate experiments.

intratumoral injections (Figure 2). We chose to use multiple injections into four quadrants of the tumors in order to maximize distribution of the virus as we had concerns about possible physical extracellular matrix barriers within the tumor that might prevent the spread of infection. VSV23 resulted in a decrease in tumor size from the initial measurement during the first 5 days after the initial treatment. After 6 days, tumors treated with VSV23 increased in size, though they remained significantly smaller than mock treated tumors after 14 days (Figure 2). Tumors treated with control viruses exhibited little to no increase in size over the first 5 days. Subsequently, these tumors increased in size and after 14 days the area was comparable to that of mock treated tumors (Figure 2). These results were generally consistent with expectations, however we had hoped to see some cases of complete tumor regression. In the case of VSV23 treated tumors we attribute the enhanced tumor destruction to the activity of IL-23, though a mechanism has not been determined at this time. It is possible that IL-23 induced increases in production of proinflammatory responses by immune cells already present in the tumor micro-environment are responsible. Such responses would include cytokines such as TNF- $\alpha$ and other anti-tumor effectors including NO. In light of the single case of temporary complete regression, we hypothesize that increasing the dose of virus and the number of treatments will result in complete tumor regression and we plan to carry out these studies in the future. Another option to increase the invasiveness of VSV23 infection in solid tumors is to increase the vascular "leakiness" of the target. Work conducted with Sindbis has recently shown the efficacy of this approach. ${ }^{49}$

The results of the CTL assay showed that mice bearing JC tumors were mounting immune responses (specifically JC targeted $\mathrm{CD}^{+} \mathrm{T}$ cells) against the cancer cells. While we did not expect to see any differences in $\mathrm{CD}^{+} \mathrm{T}$ cell recruitment (IL-23 is known to increase the effectiveness, but not the recruitment of $\mathrm{CD} 8^{+} \mathrm{T}$ cells) we hypothesized that there might be detectable differences in other immune cells populations present within tumors. ${ }^{44}$ In order to explore this idea we utilized immunohistochemistry and confocal microscopy to visualize $\mathrm{CD} 8^{+} \mathrm{T}$ cells, $\mathrm{CD} 4^{+} \mathrm{T}$ cells, macrophages, and neutrophils that had infiltrated tumors 14 days after viral treatment was started (Figure 3). The results of these experiments indicated that all four cell types were present within the tumors. This experiment was, admittedly, a qualitative one. In the future, we plan to conduct a quantitative experiment using FACS analysis of tumor homogenates to determine if there are differences in quantity of each cell type in tumors as a result of vIL-23 activity. As previously stated, we would not expect a difference in $\mathrm{CD} 8^{+} \mathrm{T}$ cell populations, but there might be increased recruitment of macrophages and $\mathrm{CD} 4^{+} \mathrm{T}$ cells.

Part of our initial hypothesis was that vIL-23 would enhance bystander effects against uninfected JC tumor cells. The differences in tumor growth between VSV23 and control virus-infected tumors supported this hypothesis. To determine the mechanistic cause of the reduction in tumor growth, we conducted a CTL assay with splenocytes harvested from animals bearing solid JC tumors, both treated and untreated. 
Due to adaptive immune responses against VSV23, the virus is no longer present in these tumors and CTL responses can be attributed to tumor cell recognition. Splenocytes were coincubated in vitro with the target JC cells, and T-cell mediated cell killing was determined by colorimetric assay. We found that lymphocytes isolated from animals bearing VSV23-treated tumors exhibited statistically significant increases in T-cell mediated lysis of JC cells when compared to cells taken from control-treated or mock-treated animals. Interestingly, there was a detectable basal level of lytic activity from splenocytes obtained from animals bearing mock-treated tumors that was indistinguishable from VSVST and VSVXN2 treated animals (Figure 4). The background killing may have been due to natural killer cells, since we did not examine lysis on YAC-1 cells or deplete the effector populations with anti-CD3 antibody. We interpret these results to indicate that while mice are capable of mounting an adaptive immune response against JC tumors, vIL-23 significantly enhances this ability. Additionally, enhanced CTL responses may be at least partially responsible for the overall decrease in tumor growth rate in VSV23 treated tumors. These data are in accordance with published data attributing the enhanced effectiveness of $\mathrm{CD} 8^{+} \mathrm{T}$ cells to upregulation of TNF- $\alpha$ by IL-23. ${ }^{44}$ It should be noted that this data also indicated maximum effectiveness of CTL cells 20 after treatment. As our tumors were harvested 14 days after treatment it is possible that maximum responses had yet to be induced. It is therefore possible that at later time points even greater CTL responses would be detected that would then be significant when compared to those in control virus-treated animals.

The limits of viral treatments are well known: host adaptive responses will eventually result in viral clearance and limit the time frame for effective tumor destruction. Here we have presented data that indicate the potential for using recombinant VSV expressing IL-23 as an oncolytic agent. Initial decreases in mammary-derived solid tumor size, and subsequent decreased growth rates due to enhanced memory responses against tumor, indicate that VSV23 is effective both before and after adaptive immune responses eliminate the viral infection from the tumor. Further studies are necessary to further elucidate the mechanisms of enhanced tumor destruction mediated by VSV23. Additionally, further studies are needed to determine whether VSV23 alone can induce permanent JC tumor regression, and if so if there is a permanent immunity to the tumor as determined by repeated JC cell challenge, and what the efficacy of VSV23 treatment of non-immunogenic and metastasizing tumors is. VSV23 may be an important addition to the tool-kit of oncolytic viruses, and may serve among sequential different viruses in treatment of solid tumors.

\section{Acknowledgments}

We would like to thank Drs Savio Woo (Mt Sinai School of Medicine), Adolfo Garcia-Sastre (Mt Sinai School of Medicine), and Daniel Meruelo (NYU School of Medicine) for their expert advice. This work could not have been accomplished without funding from the NIH to CSR, NS039746. The work described in this manuscript has been submitted to the US Patent and Trade Office, US Provisional Patent Application Serial \# 61/187,125.

\section{Disclosure}

The authors report no conflicts of interest in this work.

\section{References}

1. Jemal A, Murray T, Ward E, et al. Cancer statistics, 2005. CA Cancer J Clin. 2005;55:10-30.

2. Tseng JC, Levin B, Hurtado A, et al. Systemic tumor targeting and killing by Sindbis viral vectors. Nat Biotechnol. 2004;22:70-77.

3. Vigil A, Park MS, Martinez O, et al. Use of reverse genetics to enhance the oncolytic properties of newcastle disease virus. Cancer Res. 2007;67:8285-8292.

4. Hu JCC, Coffin RS, Davis CJ, et al. A phase I study of OncoVEX(GMCSF), a second-generation oncolytic herpes simplex virus expressing granulocyte macrophage colony-stimulating factor. Clin Cancer Res. 2006;12:6737-6747.

5. Barber GN. Vesicular stomatitis virus as an oncolytic vector. Viral Immunol. 2004;17:516-527.

6. Stojdl DF, Lichty B, Knowles S, et al. Exploiting tumor-specific defects in the interferon pathway with a previously unknown oncolytic virus. Nat Med. 2000;6:821-825.

7. Ebert O, Harbaran S, Shinozaki K, Woo SL. Systemic therapy of experimental breast cancer metastases by mutant vesicular stomatitis virus in immune-competent mice. Cancer Gene Ther. 2005;12:350-358

8. Huang TG, Ebert O, Shinozaki K, Garcia-Sastre A, Woo SLC. Oncolysis of hepatic metastasis of colorectal cancer by recombinant vesicular stomatitis virus in immune-competent mice. Mol Ther. 2003;8:434-440.

9. Shi W, Tang QQ, Chen XC, et al. Antitumor and antimetastatic activities of vesicular stomatitis virus matrix protein in a murine model of breast cancer. J Mol Med. 2009;87:493-506.

10. Gao Y, Whitaker-Dowling P, Griffin JA, Barmada MA, Bergman I. Recombinant vesicular stomatitis virus targeted to Her $2 /$ neu combined with anti-CTLA4 antibody eliminates implanted mammary tumors. Cancer Gene Ther. 2009;16:44-52.

11. Balachandran S, Porosnicu M, Barber GN. Oncolytic activity of vesicular stomatitis virus is effective against tumors exhibiting aberrant $\mathrm{p} 53$, Ras, or Myc function and involves the induction of apoptosis. J Virol. 2001;75:3474-3479.

12. Huszthy PC, Giroglou T, Tsinkalovsky O, et al. Remission of invasive, cancer stem-like glioblastoma xenografts using lentiviral vector-mediated suicide gene therapy. PLoS One. 2009;4:e6314.

13. Tumilasci VF, Oliere S, Nguyen TLA, Shamy A, Bell J, Hiscott J. Targeting the apoptotic pathway with BCL-2 inhibitors sensitizes primary chronic lymphocytic leukemia cells to vesicular stomatitis virus-induced oncolysis. J Virol. 2008;82:8487-8499. 
14. Cesaire R, Oliere S, Sharif-Askari E, et al. Oncolytic activity of vesicular stomatitis virus in primary adult T-cell leukemia. Oncogene. 2006;25:349-358.

15. Shinozaki K, Ebert O, Woo SLC. Treatment of multi-focal colorectal carcinoma metastatic to the liver of immune-competent and syngeneic rats by hepatic artery infusion of oncolytic vesicular stomatitis virus. Int J Cancer. 2005;114:659-664.

16. Shinozaki K, Ebert O, Kournioti C, Tai YS, Woo SLC. Oncolysis of multifocal hepatocellular carcinoma in the rat liver by hepatic artery infusion of vesicular stomatitis virus. Mol Ther. 2004;9:368-376.

17. Altomonte J, Braren R, Schulz S, et al. Synergistic antitumor effects of transarterial viroembolization for multifocal hepatocellular carcinoma in rats. Hepatology. 2008;48:1864-1873.

18. Feng Y, He G, Wu Y, Wen Y. [Effect of apoptosis of vesicular stomatitis virus on tumor cells of nasopharyngeal carcinoma in vivo]. Lin Chung Er Bi Yan Hou Tou Jing Wai Ke Za Zhi. 2008;22:995-997.

19. Hadaschik BA, Zhang K, So AI, et al. Oncolytic vesicular stomatitis viruses are potent agents for intravesical treatment of high-risk bladder cancer. Cancer Res. 2008;68:4506-4510.

20. Hadaschik BA, Zhang K, So AI, et al. Oncolytic vesicular stomatitis viruses as intravesical agents against non-muscle-invasive bladder cancer. Urologe. 2008;47:1145-1151.

21. Balachandran S, Barber GN. Vesicular stomatitis virus (VSV) therapy of tumors. IUBMB Life. 2000;50:135-138.

22. Johnson KM, Vogel JE, Peralta PH. Clinical and serological response to laboratory-acquired human infection by Indiana type vesicular stomatitis virus (VSV). Am J Trop Med Hyg. 1966;15:244-246.

23. Fields BN, Hawkins K. Human infection with the virus of vesicular stomatitis during an epizootic. N Engl J Med. 1967;277:989-994.

24. Rose JK, Whitt MA. Rhabdoviridae: the viruses and their replication. In: Knipe DM, P.M. H, eds. Fields Virology. Philadelphia, PA: Lippincott Williams and Wilkins, 2001:1221-1242.

25. Ebert O, Shinozaki K, Kournioti C, Park MS, Garcia-Sastre A, Woo SLc. Syncytia induction enhances the oncolytic potential of vesicular stomatitis virus in virotherapy for cancer. Cancer Res. 2004;9:S397-S397.

26. Shin EJ, Wanna GB, Choi B, et al. Interleukin-12 expression enhances vesicular stomatitis virus oncolytic therapy in murine squamous cell carcinoma. Laryngoscope. 2007;117:210-214.

27. Wollmann G, Robek MD, van den Pol AN. Variable deficiencies in the interferon response enhance susceptibility to vesicular stomatitis virus oncolytic actions in glioblastoma cells but not in normal human glial cells. J Virol. 2007;81:1479-1491.

28. Connor JH, Naczki C, Koumenis C, Lyles DS. Replication and cytopathic effect of oncolytic vesicular stomatitis virus in hypoxic tumor cells in vitro and in vivo. J Virol. 2004;78:8960-8970.

29. Iwaki H, Barnavon Y, Bash JA, Wallack MK. Vaccinia virus-infected C-C36 colon-tumor cell lysates stimulate cellular-responses in vitro and protect syngeneic balb/c mice from tumor-cell challenge. J Surg Oncol. 1989;40:90-96.

30. Liu L, Chavan R, Feinberg MB. Dendritic cells are preferentially targeted among hematolymphocytes by modified vaccinia virus Ankara and play a key role in the induction of virus-specific $\mathrm{T}$ cell responses in vivo. BMC Immunol. 2008;9:15.
31. Kawai T, Akira S. Innate immune recognition of viral infection. Nat Immunol. 2006; 7:131-137.

32. Rowland-Jones S, Dong T. Dying T cells trigger autoimmunity in HIV. Nat Med. 2007;13:1413-1415.

33. Parham C, Chirica M, Timans J, et al. A receptor for the heterodimeric cytokine IL-23 is composed of IL-12Rbeta1 and a novel cytokine receptor subunit, IL-23R. J Immunol. 2002;168:5699-708.

34. Bastos KRB, Marinho CRF, Barboza R, Russo M, Alvarez JM, Lima MRD. What kind of message does IL-12/IL-23 bring to macrophages and dendritic cells? Microbes Infect. 2004;6:630-636.

35. Ivanov II, McKenzie BS, Zhou L, et al. The orphan nuclear receptor ROR gamma $t$ directs the differentiation program of proinflammatory IL-17(+) T helper cells. Cell. 2006;126:1121-1133.

36. Oppmann B, Lesley R, Blom B, et al. Novel p19 protein engages IL-12p40 to form a cytokine, IL-23, with biological activities similar as well as distinct from IL-12. Immunity. 2000;13:715-725.

37. McKenzie BS, Kastelein RA, Cua DJ. Understanding the IL-23-IL-17 immune pathway. Trends Immunol. 2006;27:17-23.

38. Kaiga T, Sato M, Kaneda H, Iwakura Y, Takayama T, Tahara H. Systemic administration of IL-23 induces potent antitumor immunity primarily mediated through Th1-type response in association with the endogenously expressed IL-12. J Immunol. 2007;178:7571-7580.

39. Tahara H, Lotze MT. Antitumor effects of interleukin-12 (IL-12): applications for the immunotherapy and gene therapy of cancer. Gene Ther. 1995;2:96-106.

40. Nastala CL, Edington HD, McKinney TG, et al. Recombinant IL-12 administration induces tumor regression in association with IFN-gamma production. J Immunol. 1994;153:1697-1706.

41. Lo $\mathrm{CH}$, Lee SC, Wu PY, et al. Antitumor and antimetastatic activity of IL-23. J Immunol. 2003;171:600-607.

42. Cohen J. IL-12 deaths: explanation and a puzzle. Science. 1995;270:908

43. Car BD, Eng VM, Lipman JM, Anderson TD. The toxicology of interleukin-12: a review. Toxicol Pathol. 1999;27:58-63.

44. Overwijk WW, de Visser KE, Tirion FH, et al. Immunological and antitumor effects of IL-23 as a cancer vaccine adjuvant. J Immunol. 2006; $176: 5213-5222$

45. Hao JS, Shan BE. Immune enhancement and anti-tumour activity of IL-23. Cancer Immunol Immunother. 2006;55:1426-1431.

46. Liu L, Shan B, Feng Y. Antitumor effects and immunoregulation mechanisms of IL-23 gene in mouse mammary cancer mediated by retrovirus. Cell Immunol. 2009;258:181-187.

47. Miller JM, Bidula SM, Jensen TM, Reiss CS. Cytokine-modified VSV is highly attenuated for neural pathology but is both highly immunogenic and oncolytic. International Journal of Interferon, Cytokine and Mediator Research. 2009;1:15-32.

48. Lawson ND, Stillman EA, Whitt MA, Rose JK. Recombinant vesicular stomatitis viruses from DNA. Proc Natl Acad Sci U S A. 1995;92:4477-4481.

49. Tseng JC, Granot T, DiGiacomo V, Levin B, Meruelo D. Enhanced specific delivery and targeting of oncolytic Sindbis viral vectors by modulating vascular leakiness in tumor. Cancer Gene Ther. 2010;17:244-255

International Journal of Interferon, Cytokine and Mediator Research

Dovepress

\section{Publish your work in this journal}

The International Journal of Interferon, Cytokine and Mediator Research is an international, peer-reviewed, open-access, online journal. The focus of the journal is to publish original research, reports, editorials, reviews and commentaries on all aspects of interferon, cytokine and mediators of inflammation from labora- tory science to therapeutic indications and clinical studies. The manuscript management system is completely online and includes a very quick and fair peer-review system, which is all easy to use. Visit http://www.dovepress.com/testimonials.php to read real quotes from published authors. 